\title{
Matrix Cauchy and Hilbert transforms in Hermitean quaternionic Clifford analysis
}

\author{
Ricardo Abreu-Blaya Juan Bory-Reyes Fred Brackx \\ Hennie De Schepper Frank Sommen
}

\begin{abstract}
Recently the basic setting has been established for the development of quaternionic Hermitean Clifford analysis, a theory centred around the simultaneous null solutions, called q-Hermitean monogenic functions, of four Hermitean Dirac operators in a quaternionic Clifford algebra setting. Borel-Pompeiu and Cauchy integral formulae have been established in this framework by means of a $(4 \times 4)$ circulant matrix approach. By means of the matricial quaternionic Hermitean Cauchy kernel involved in these formulae, a quaternionic Hermitean Cauchy integral may be defined. The subsequent study of the boundary limits of this Cauchy integral then leads to the definition of a quaternionic Hermitean Hilbert transform. These integral transforms are studied in the present paper.
\end{abstract}

Keywords. Quaternionic Hermitean Clifford analysis, Cauchy integral, Hilbert transform.

Mathematics Subject Classification (2000). 30G35.

\section{Introduction}

Euclidean Clifford analysis is a higher dimensional function theory offering a refinement of classical harmonic analysis. The theory is centred around the concept of monogenic functions, i.e. null solutions of a first order vector valued rotation invariant differential operator, called Dirac operator, which factorizes the Laplacian; monogenic functions may thus also be seen as a generalization of holomorphic functions in the complex plane. Its roots go back as early as the 1930's. For more details on this function theory we refer e.g. to the standard references $[3,10,12,13,14]$.

More recently Hermitean Clifford analysis emerged as a refinement of the Euclidean setting for the case of $\mathbb{R}^{2 n}$. Here, Hermitean monogenic functions are 
considered, i.e. functions taking values either in a complex Clifford algebra or in complex spinor space, which are simultaneous null solutions of two complex Hermitean Dirac operators, which are invariant under the action of the unitary group. For the systematic development of this function theory we refer e.g. to $[4,5,6]$.

In the recent papers $[8,9,11,16]$, the Hermitean Clifford analysis setting was further generalized by considering functions on $\mathbb{R}^{4 n}$ with values in a quaternionic Clifford algebra. Here the so-called quaternionic Hermitean Dirac equation is studied, which corresponds to functions being simultaneous null solutions of four mutually related quaternionic Dirac operators, which are invariant under the action of the symplectic group. In [1], Borel-Pompeiu and Cauchy integral formulas are established in this quaternionic Hermitean setting, by following a $(4 \times 4)$ circulant matrix approach, which can be seen as similar in spirit to the circulant $(2 \times 2)$ matrix approach introduced in [7] within the complex Hermitean Clifford case. It is clear that, by means of the matricial quaternionic Hermitean Cauchy kernel defined in that paper, also a quaternionic Hermitean Cauchy integral may be defined, the study of the boundary limits of which will subsequently lead to the introduction of a quaternionic Hermitean Hilbert transform. The study of these integral transforms is the main subject of the present paper.

\section{Preliminaries}

Let $\mathbb{R}_{0, m}$ be the real Clifford algebra of signature $(0, m)$ constructed over the Euclidean space $\mathbb{R}^{m}$, which is endowed with the orthonormal basis $\left(e_{1}, \ldots, e_{m}\right)$. The non-commutative multiplication in $\mathbb{R}_{0, m}$ then is governed by the rules

$$
e_{\ell}^{2}=-1, \ell=1, \ldots, m, \quad e_{\ell} e_{k}+e_{k} e_{\ell}=0, \ell, k=1, \ldots, m, \ell \neq k
$$

and the following automorphisms are considered:

(i) the conjugation, given by $\bar{e}_{\ell}=-e_{\ell}$ and $\overline{a b}=\bar{b} \bar{a}$, for any $a, b \in \mathbb{R}_{0, m}$;

(ii) the main involution, given by $\widetilde{e}_{\ell}=-e_{\ell}$ and $\widetilde{a b}=\widetilde{a} \widetilde{b}$ for any $a, b \in \mathbb{R}_{0, m}$.

In particular we consider the skew-field of quaternions $\mathbb{H}$ whose elements will be denoted by $q=x_{0}+i x_{1}+j x_{2}+k x_{3}$ with $i^{2}=j^{2}=k^{2}=-1$ and $i j=$ $-j i=k$. Clearly $\mathbb{H}$ may be identified with the Clifford algebra $\mathbb{R}_{0,2}$ making the identifications $i \leftrightarrow e_{1}, j \leftrightarrow e_{2}$ and $k \leftrightarrow e_{1} e_{2}$. The automorphisms (i) and (ii) then respectively lead to the $\mathbb{H}$-conjugation

$$
\bar{q}=x_{0}-i x_{1}-j x_{2}-k x_{3}
$$

and to the main $\mathbb{H}$-involution

$$
q^{\gamma} \equiv \tilde{q}=x_{0}-i x_{1}-j x_{2}+k x_{3}
$$


However, it is quite natural to introduce two more $\mathbb{H}$-involutions defined by

$$
\begin{aligned}
& q^{\alpha}=x_{0}+i x_{1}-j x_{2}-k x_{3} \\
& q^{\beta}=x_{0}-i x_{1}+j x_{2}-k x_{3}
\end{aligned}
$$

In what follows we will consider the Clifford algebra $\mathbb{H}_{m}=\mathbb{H} \otimes_{\mathbb{R}} \mathbb{R}_{0, m}$, with elements $\lambda=\sum_{A} e_{A} \lambda_{A}, \lambda_{A} \in \mathbb{H}$, where $e_{A}$ are the canonical basis elements of $\mathbb{R}_{0, m}$ generated by $\left(e_{1}, \ldots, e_{m}\right)$, and where it is tacitly understood that the quaternionic scalars commute with the Clifford basis vectors. The quaternionic Hermitean conjugate of $\lambda \in \mathbb{H}_{m}$ is defined as the composition of the $\mathbb{H}$-conjugation and the Clifford conjugation in $\mathbb{R}_{0, m}$, i.e. $\lambda^{\dagger}=\sum_{A} \overline{e_{A}} \overline{\lambda_{A}}$. We also define a norm in $\mathbb{H}_{m}$, namely

$$
|\lambda|=\left[\lambda \lambda^{\dagger}\right]_{0}=\sum_{A}\left|\lambda_{A}\right|^{2}
$$

where $[\cdot]_{0}$ stands for the scalar part of a quaternionic Clifford number.

From now on we assume the dimension to be a quadruple: $m=4 n$, and we introduce an alternative basis for $\mathbb{H}_{4 n}$.

Definition 1 ([16]) The quaternionic Witt basis of $\mathbb{H}_{4 n}=\mathbb{H} \otimes_{\mathbb{R}} \mathbb{R}_{0,4 n}$ is given by $\left\{f_{\ell}, f_{\ell}^{\alpha}, f_{\ell}^{\beta}, f_{\ell}^{\gamma}\right\}, \ell=1, \ldots, n$, where

$$
\begin{aligned}
& f_{\ell}=e_{1+4(\ell-1)}-i e_{2+4(\ell-1)}-j e_{3+4(\ell-1)}-k e_{4+4(\ell-1)}, \\
& f_{\ell}^{\alpha}=e_{1+4(\ell-1)}-i e_{2+4(\ell-1)}+j e_{3+4(\ell-1)}+k e_{4+4(\ell-1)}, \\
& f_{\ell}^{\beta}=e_{1+4(\ell-1)}+i e_{2+4(\ell-1)}-j e_{3+4(\ell-1)}+k e_{4+4(\ell-1)}, \\
& f_{\ell}^{\gamma}=e_{1+4(\ell-1)}+i e_{2+4(\ell-1)}+j e_{3+4(\ell-1)}-k e_{4+4(\ell-1)} .
\end{aligned}
$$

Let us now define the following real Clifford vectors associated to an element $\left(x_{1}, \ldots, x_{4 n}\right)$ in $\mathbb{R}^{4 n}$ :

$$
\begin{aligned}
\underline{X}=\underline{X}_{0} & =\sum_{\ell=1}^{n}\left(e_{4 \ell-3} x_{4 \ell-3}+e_{4 \ell-2} x_{4 \ell-2}+e_{4 \ell-1} x_{4 \ell-1}+e_{4 \ell} x_{4 \ell}\right) \\
\underline{X}_{1} & =\sum_{\ell=1}^{n}\left(e_{4 \ell-3} x_{4 \ell-2}-e_{4 \ell-2} x_{4 \ell-3}-e_{4 \ell-1} x_{4 \ell}+e_{4 \ell} x_{4 \ell-1}\right) \\
\underline{X}_{2} & =\sum_{\ell=1}^{n}\left(e_{4 \ell-3} x_{4 \ell-1}+e_{4 \ell-2} x_{4 \ell}-e_{4 \ell-1} x_{4 \ell-3}-e_{4 \ell} x_{4 \ell-2}\right) \\
\underline{X}_{3} & =\sum_{\ell=1}^{n}\left(e_{4 \ell-3} x_{4 \ell}-e_{4 \ell-2} x_{4 \ell-1}+e_{4 \ell-1} x_{4 \ell-2}-e_{4 \ell} x_{4 \ell-3}\right)
\end{aligned}
$$


Here $\underline{X}_{0}$ corresponds to the usual Clifford vector $\underline{X}$ associated to $\left(x_{1}, \ldots, x_{4 n}\right)$, while $\underline{X}_{1}, \underline{X}_{2}, \underline{X}_{3}$ are similar to the twisted vector variable of the complex Hermitean case, see $[6,4]$. We have $\underline{X}_{0}^{2}=\underline{X}_{1}^{2}=\underline{X}_{2}^{2}=\underline{X}_{3}^{2}=-|\underline{X}|^{2}$, while

$$
\left\{\underline{X}_{r}, \underline{X}_{s}\right\} \equiv \underline{X}_{r} \underline{X}_{s}+\underline{X}_{s} \underline{X}_{r}=0, \quad r, s=0,1,2,3, r \neq s
$$

Next we define the differential operators

$$
\begin{aligned}
\partial_{\underline{X}}=\partial_{\underline{X}_{0}} & =\sum_{\ell=1}^{n}\left(e_{4 \ell-3} \partial_{x_{4 \ell-3}}+e_{4 \ell-2} \partial_{x_{4 \ell-2}}+e_{4 \ell-1} \partial_{x_{4 \ell-1}}+e_{4 \ell} \partial_{x_{4 \ell}}\right) \\
\partial_{\underline{X}_{1}} & =\sum_{\ell=1}^{n}\left(e_{4 \ell-3} \partial_{x_{4 \ell-2}}-e_{4 \ell-2} \partial_{x_{4 \ell-3}}-e_{4 \ell-1} \partial_{x_{4 \ell}}+e_{4 \ell} \partial_{x_{4 \ell-1}}\right) \\
\partial_{\underline{X}_{2}} & =\sum_{\ell=1}^{n}\left(e_{4 \ell-3} \partial_{x_{4 \ell-1}}+e_{4 \ell-2} \partial_{x_{4 \ell}}-e_{4 \ell-1} \partial_{x_{4 \ell-3}}-e_{4 \ell} \partial_{x_{4 \ell-2}}\right) \\
\partial_{\underline{X}_{3}} & =\sum_{\ell=1}^{n}\left(e_{4 \ell-3} \partial_{x_{4 \ell}}-e_{4 \ell-2} \partial_{x_{4 \ell-1}}+e_{4 \ell-1} \partial_{x_{4 \ell-2}}-e_{4 \ell} \partial_{x_{4 \ell-3}}\right)
\end{aligned}
$$

Again, note that $\partial_{\underline{X}_{0}}$ corresponds to the usual Dirac operator $\partial_{\underline{X}}$, while $\partial_{\underline{X}_{1}}, \partial_{\underline{X}_{2}}$, $\partial_{\underline{X}_{3}}$ are analogues of the twisted Dirac operator in the complex Hermitean case. Similarly as above, we have the relations $\partial_{\underline{X}_{0}}^{2}=\partial_{\underline{X}_{1}}^{2}=\partial_{\underline{X}_{2}}^{2}=\partial_{\underline{X}_{3}}^{2}=-\Delta_{4 n}$, and

$$
\left\{\partial_{\underline{X}_{r}}, \partial_{\underline{X}_{s}}\right\} \equiv \partial_{\underline{X}_{r}} \partial_{\underline{X}_{s}}+\partial_{\underline{X}_{s}} \partial_{\underline{X}_{r}}=0, \quad r, s=0,1,2,3, r \neq s
$$

Starting from these Euclidean variables and operators, their quaternionic Hermitean counterparts are defined. First the quaternionic Hermitean variables are given by

$$
\begin{aligned}
\underline{Z}=\underline{Z}_{0} & =\underline{X}_{0}+i \underline{X}_{1}+j \underline{X}_{2}+k \underline{X}_{3} \\
\underline{Z}_{1} & =\underline{X}_{0}+i \underline{X}_{1}-j \underline{X}_{2}-k \underline{X}_{3} \\
\underline{Z}_{2} & =\underline{X}_{0}-i \underline{X}_{1}+j \underline{X}_{2}-k \underline{X}_{3} \\
\underline{Z}_{3} & =\underline{X}_{0}-i \underline{X}_{1}-j \underline{X}_{2}+k \underline{X}_{3}
\end{aligned}
$$

or equivalently, in terms of the quaternionic Witt basis elements, by

$$
\begin{aligned}
\underline{Z}=\underline{Z}_{0} & =\sum_{\ell=1}^{n} f_{\ell}\left(x_{4 \ell-3}+i x_{4 \ell-2}+j x_{4 \ell-1}+k x_{4 \ell}\right) \\
\underline{Z}_{1} & =\sum_{\ell=1}^{n} f_{\ell}^{\alpha}\left(x_{4 \ell-3}+i x_{4 \ell-2}-j x_{4 \ell-1}-k x_{4 \ell}\right) \\
\underline{Z}_{2} & =\sum_{\ell=1}^{n} f_{\ell}^{\beta}\left(x_{4 \ell-3}-i x_{4 \ell-2}+j x_{4 \ell-1}-k x_{4 \ell}\right) \\
\underline{Z}_{3} & =\sum_{\ell=1}^{n} f_{\ell}^{\gamma}\left(x_{4 \ell-3}-i x_{4 \ell-2}-j x_{4 \ell-1}+k x_{4 \ell}\right)
\end{aligned}
$$


We have the basic relations

$$
\underline{Z}_{0} \underline{Z}_{0}^{\dagger}+\underline{Z}_{1} \underline{Z}_{1}^{\dagger}+\underline{Z}_{2} \underline{Z}_{2}^{\dagger}+\underline{Z}_{3} \underline{Z}_{3}^{\dagger}=\underline{Z}_{0}^{\dagger} \underline{Z}_{0}+\underline{Z}_{1}^{\dagger} \underline{Z}_{1}+\underline{Z}_{2}^{\dagger} \underline{Z}_{2}+\underline{Z}_{3}^{\dagger} \underline{Z}_{3}=16|\underline{X}|^{2}
$$

The Hermitean Dirac operators are similarly derived from the Euclidean ones:

$$
\begin{aligned}
\partial_{\underline{Z}_{0}} & =\frac{1}{16}\left(\partial_{\underline{X}_{0}}+i \partial_{\underline{X}_{1}}+j \partial_{\underline{X}_{2}}+k \partial_{\underline{X}_{3}}\right) \\
\partial_{\underline{Z}_{1}} & =\frac{1}{16}\left(\partial_{\underline{X}_{0}}+i \partial_{\underline{X}_{1}}-j \partial_{\underline{X}_{2}}-k \partial_{\underline{X}_{3}}\right) \\
\partial_{\underline{Z}_{2}} & =\frac{1}{16}\left(\partial_{\underline{X}_{0}}-i \partial_{\underline{X}_{1}}+j \partial_{\underline{X}_{2}}-k \partial_{\underline{X}_{3}}\right) \\
\partial_{\underline{Z}_{3}} & =\frac{1}{16}\left(\partial_{\underline{X}_{0}}-i \partial_{\underline{X}_{1}}-j \partial_{\underline{X}_{2}}+k \partial_{\underline{X}_{3}}\right)
\end{aligned}
$$

They establish a decomposition of the Laplacian in $\mathbb{R}^{4 n}$ :

$$
\frac{1}{16} \Delta_{4 n}=\partial_{\underline{Z}_{0}} \partial_{\underline{Z}_{0}}^{\dagger}+\partial_{\underline{Z}_{1}} \partial_{\underline{Z}_{1}}^{\dagger}+\partial_{\underline{Z}_{2}} \partial_{\underline{Z}_{2}}^{\dagger}+\partial_{\underline{Z}_{3}} \partial_{\underline{Z}_{3}}^{\dagger}=\partial_{\underline{Z}_{0}}^{\dagger} \partial_{\underline{Z}_{0}}+\partial_{\underline{Z}_{1}}^{\dagger} \partial_{\underline{Z}_{1}}+\partial_{\underline{Z}_{2}}^{\dagger} \partial_{\underline{Z}_{2}}+\partial_{\underline{Z}_{3}}^{\dagger} \partial_{\underline{Z}_{3}}
$$

Finally we introduce the central concept in our setting, see [16].

Definition 2 Let $\Omega$ be an open set in $\mathbb{R}^{4 n}$. A continuously differentiable function $f: \Omega \mapsto \mathbb{H}_{4 n}$ is said to be q-Hermitean monogenic in $\Omega$ iff it satisfies the system $\partial_{\underline{Z}_{0}} f=\partial_{\underline{Z}_{1}} f=\partial_{\underline{Z}_{2}} f=\partial_{\underline{Z}_{3}} f=0$, or, equivalently, the system $\partial_{\underline{X}_{0}} f=\partial_{\underline{X}_{1}} f=$ $\partial_{\underline{X}_{2}} f=\partial_{\underline{X}_{3}} f=0$.

A $q$-Hermitean monogenic function in $\Omega$ is automatically monogenic in $\Omega$ and thus harmonic in $\Omega$. It is worth mentioning that in [11], the author focussed on the underlying group invariance properties and for that purpose introduced a different system. However, it was proven in detail in [8] that the system in Definition 2, introduced first in [16], and the system in Definition 9 of [11] are equivalent, giving rise to the same notion of quaternionic Hermitean monogenic functions.

The fundamental solutions of the Dirac operators $\partial_{\underline{X}_{r}}, r=0,1,2,3$, i.e. the orthogonal Cauchy kernels, are respectively given by

$$
E_{r}(\underline{X})=-\frac{1}{a_{4 n}} \frac{\underline{X}_{r}}{|\underline{X}|^{4 n}}, \quad r=0,1,2,3
$$

where $a_{4 n}$ denotes the area of the unit sphere $S^{4 n-1}$ in $\mathbb{R}^{4 n}$. Explicitly, this means that $\partial_{\underline{X}_{r}} E_{r}(\underline{X})=\delta(\underline{X}), r=0,1,2,3$, where $\delta(\underline{X})$ stands for the delta distribution in $\mathbb{R}^{4 n}$. Similarly as above, we now introduce the Hermitean Cauchy kernels by

$$
\begin{aligned}
& \mathcal{E}_{0}=E_{0}-i E_{1}-j E_{2}-k E_{3} \\
& \mathcal{E}_{1}=E_{0}-i E_{1}+j E_{2}+k E_{3} \\
& \mathcal{E}_{2}=E_{0}+i E_{1}-j E_{2}+k E_{3}
\end{aligned}
$$




$$
\mathcal{E}_{3}=E_{0}+i E_{1}+j E_{2}-k E_{3}
$$

Explicitly this yields

$$
\mathcal{E}_{r}(\underline{Z})=\frac{1}{a_{4 n}} \frac{\underline{Z}_{r}^{\dagger}}{|\underline{Z}|^{4 n}}, \quad r=0,1,2,3
$$

The kernels $\mathcal{E}_{r}$ are not the fundamental solutions of the respective Hermitean Dirac operators $\partial_{\underline{Z}_{r}}, r=0,1,2,3$. However, the following result holds, see [1].

Theorem 1 Introducing the circulant $(4 \times 4)$ matrices

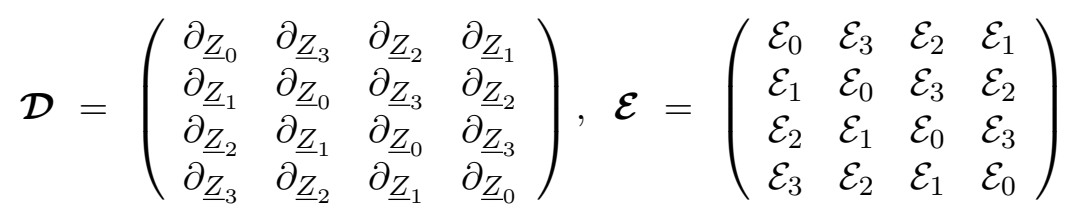

and

$$
\boldsymbol{\delta}=\left(\begin{array}{llll}
\delta & 0 & 0 & 0 \\
0 & \delta & 0 & 0 \\
0 & 0 & \delta & 0 \\
0 & 0 & 0 & \delta
\end{array}\right)
$$

one obtains that $\mathcal{D}^{T} \mathcal{E}=\mathcal{E} \mathcal{D}^{T}=\boldsymbol{\delta}$.

Therefore, $\mathcal{E}$ may be considered as a fundamental solution of $\mathcal{D}$, when this concept is reinterpreted in a matricial context. This simple but remarkable fact has lead to the idea of following a matrix approach in order to establish a Cauchy integral formula in the quaternionic Hermitean setting, see [1]. In the same order of ideas, we will now use the kernel $\mathcal{E}$ to define Cauchy and Hilbert transforms in the matricial quaternionic Hermitean setting.

To this end we associate, with functions $g_{0}, g_{1}, g_{2}$ and $g_{3}$ defined in $\Omega \subset \mathbb{R}^{4 n}$ and taking values in $\mathbb{H}_{4 n}$, the $(4 \times 4)$ circulant matrix function

$$
\boldsymbol{G}=\left(\begin{array}{cccc}
g_{0} & g_{3} & g_{2} & g_{1} \\
g_{1} & g_{0} & g_{3} & g_{2} \\
g_{2} & g_{1} & g_{0} & g_{3} \\
g_{3} & g_{2} & g_{1} & g_{0}
\end{array}\right) \equiv \operatorname{circ}\left(\begin{array}{c}
g_{0} \\
g_{1} \\
g_{2} \\
g_{3}
\end{array}\right)
$$

the latter notation being justified by the fact that a circulant matrix is fully determined by its first column. In what follows, we will use small letters to denote $\mathbb{H}_{4 n}$-valued functions and boldface capitals for $(4 \times 4)$ circulant matrix functions. We say that $\boldsymbol{G}$ belongs to some class of functions if all its entries belong to that class. In particular, the spaces of $k$-times continuously differentiable, of $\alpha$-Hölder continuous $(0<\alpha \leq 1)$ and of $p$-integrable $(4 \times 4)$ circulant matrix functions on some suitable subset $\mathbf{E}$ of $\mathbb{R}^{4 n}$ are respectively denoted by $\mathbf{C}^{k}(\mathbf{E}), \mathbf{C}^{0, \alpha}(\mathbf{E})$ and 
$\mathbf{L}^{p}(\mathbf{E})$. The corresponding spaces of $\mathbb{H}_{4 n}$-valued functions are, as usual, denoted by $C^{k}(\mathbf{E}), C^{0, \alpha}(\mathbf{E})$ and $L^{p}(\mathbf{E})$. Moreover, introducing the non-negative function $\|\boldsymbol{G}(\underline{X})\|=\max _{r=0,1,2,3}\left\{\left|g_{r}(\underline{X})\right|\right\}$, the classes $\mathbf{C}^{0, \alpha}(\mathbf{E})$ and $\mathbf{L}^{p}(\mathbf{E})$ may also be defined by means of the respective traditional conditions

$$
\|\boldsymbol{G}\|_{\alpha}=\max _{\underline{X} \in \mathbf{E}}\|\boldsymbol{G}(\underline{X})\|+\sup _{\underline{X}, \underline{Y} \in \mathbf{E}, \underline{X} \neq \underline{Y}} \frac{\|\boldsymbol{G}(\underline{X})-\boldsymbol{G}(\underline{Y})\|}{|\underline{X}-\underline{Y}|^{\alpha}}<+\infty
$$

and

$$
\|\boldsymbol{G}\|_{p}=\left(\int_{\mathbf{E}}\|\boldsymbol{G}(\underline{X})\|^{p}\right)^{\frac{1}{p}}<+\infty
$$

Definition 3 We call the $(4 \times 4)$ circulant matrix function $\boldsymbol{G}$ (left) $\mathbf{Q}-$ Hermitean monogenic in $\Omega$ if and only if, in $\Omega$,

$$
\mathcal{D}^{T} \boldsymbol{G}=\boldsymbol{O}
$$

where $\boldsymbol{O}$ denotes the matrix with zero entries.

The above system (3) explicitly reads

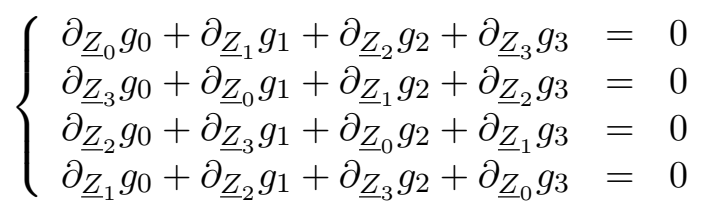

whence clearly, the $\mathbf{Q}-$ Hermitean monogenicity of $\boldsymbol{G}$ does not imply the $q$-Hermitean monogenicity of its entries. However, an important special case occurs when considering the matrix function

$$
\boldsymbol{G}_{0}=\left(\begin{array}{cccc}
g & 0 & 0 & 0 \\
0 & g & 0 & 0 \\
0 & 0 & g & 0 \\
0 & 0 & 0 & g
\end{array}\right) \equiv \operatorname{circ}\left(\begin{array}{l}
g \\
0 \\
0 \\
0
\end{array}\right)
$$

Indeed, $\boldsymbol{G}_{0}$ is $\mathbf{Q}-$ Hermitean monogenic if and only if the function $g$ is $q$-Hermitean monogenic.

\section{The Q-Hermitean Cauchy transform}

Let $\Omega$ be a bounded, simply connected domain in $\mathbb{R}^{4 n}$ with smooth boundary $\Gamma=\partial \Omega$. The unit normal vector $\underline{n}(\underline{X})$ on $\Gamma$ at a point $\underline{X} \in \Gamma$ is given by

$$
\underline{n}(\underline{X})=\underline{n}_{0}(\underline{X})=\sum_{\ell=1}^{n}\left(e_{4 \ell-3} n_{4 \ell-3}(\underline{X})+e_{4 \ell-2} n_{4 \ell-2}(\underline{X})+e_{4 \ell-1} n_{4 \ell-1}(\underline{X})+e_{4 \ell} n_{4 \ell}(\underline{X})\right)
$$


and we moreover introduce its "twisted" versions

$$
\begin{aligned}
& \underline{n}_{1}(\underline{X})=\sum_{\ell=1}^{n}\left(e_{4 \ell-3} n_{4 \ell-2}(\underline{X})-e_{4 \ell-2} n_{4 \ell-3}(\underline{X})-e_{4 \ell-1} n_{4 \ell}(\underline{X})+e_{4 \ell} n_{4 \ell-1}(\underline{X})\right) \\
& \underline{n}_{2}(\underline{X})=\sum_{\ell=1}^{n}\left(e_{4 \ell-3} n_{4 \ell-1}(\underline{X})+e_{4 \ell-2} n_{4 \ell}(\underline{X})-e_{4 \ell-1} n_{4 \ell-3}(\underline{X})-e_{4 \ell} n_{4 \ell-2}(\underline{X})\right) \\
& \underline{n}_{3}(\underline{X})=\sum_{\ell=1}^{n}\left(e_{4 \ell-3} n_{4 \ell}(\underline{X})-e_{4 \ell-2} n_{4 \ell-1}(\underline{X})+e_{4 \ell-1} n_{4 \ell-2}(\underline{X})-e_{4 \ell} n_{4 \ell-3}(\underline{X})\right)
\end{aligned}
$$

giving rise in the usual way (up to a constant factor) to their Hermitean counterparts

$$
\begin{aligned}
& \mathcal{N}_{0}=\frac{1}{16}\left(\underline{n}_{0}+i \underline{n}_{1}+j \underline{n}_{2}+k \underline{n}_{3}\right) \\
& \mathcal{N}_{1}=\frac{1}{16}\left(\underline{n}_{0}+i \underline{n}_{1}-j \underline{n}_{2}-k \underline{n}_{3}\right) \\
& \mathcal{N}_{2}=\frac{1}{16}\left(\underline{n}_{0}-i \underline{n}_{1}+j \underline{n}_{2}-k \underline{n}_{3}\right) \\
& \mathcal{N}_{3}=\frac{1}{16}\left(\underline{n}_{0}-i \underline{n}_{1}-j \underline{n}_{2}+k \underline{n}_{3}\right)
\end{aligned}
$$

and the corresponding circulant matrix

$$
\mathcal{N}=\operatorname{circ}\left(\begin{array}{c}
\mathcal{N}_{0} \\
\mathcal{N}_{1} \\
\mathcal{N}_{2} \\
\mathcal{N}_{3}
\end{array}\right)
$$

Then the following Cauchy integral formulae were established in [1] for $\mathbf{Q}-$ Hermitean monogenic matrix functions and $q$-hermitean monogenic functions, respectively.

Theorem 2 (Q-Hermitean Cauchy integral formula) Let $\Omega$ be as above. If the circulant matrix function $\boldsymbol{G}$, given by (2), belongs to $\mathbf{C}^{1}(\bar{\Omega})$ and moreover is $\mathbf{Q}-$ Hermitean monogenic in $\Omega$ then

$$
\int_{\Gamma} \mathcal{E}(\underline{Z}-\underline{V}) \mathcal{N}^{T}(\underline{Z}) \boldsymbol{G}(\underline{X}) d S(\underline{X})= \begin{cases}\boldsymbol{G}(\underline{Y}), & \underline{Y} \in \Omega^{+} \\ \boldsymbol{O}, & \underline{Y} \in \Omega^{-}\end{cases}
$$

In particular, if the function $g \in C^{1}(\bar{\Omega})$ is q-Hermitean monogenic in $\Omega$ then

$$
\int_{\Gamma} \mathcal{E}(\underline{Z}-\underline{V}) \mathcal{N}^{T}(\underline{Z}) \boldsymbol{G}_{0}(\underline{X}) d S(\underline{X})= \begin{cases}\boldsymbol{G}_{0}(\underline{Y}), & \underline{Y} \in \Omega^{+} \\ \boldsymbol{O}, & \underline{Y} \in \Omega^{-}\end{cases}
$$

where $\boldsymbol{G}_{0}$ is the corresponding matrix function (4). Here, $\underline{Z}$ and $\underline{V}$ denote the quaternionic Hermitean counterparts of the Clifford vectors $\underline{X}$ and $\underline{Y}$, respectively. 
These results motivate the introduction of the notion of a $\mathbf{Q}$-Hermitean Cauchy transform, given by

$$
\mathcal{C}[\boldsymbol{G}](\underline{Y})=\int_{\Gamma} \mathcal{E}(\underline{Z}-\underline{V}) \mathcal{N}^{T}(\underline{Z}) \boldsymbol{G}(\underline{X}) d S(\underline{X}), \quad \underline{Y} \notin \Gamma
$$

for $\boldsymbol{G} \in \mathbf{C}(\Gamma)$. It directly follows that $\mathcal{C}[\boldsymbol{G}]$ is a $\mathbf{Q}$-Hermitean monogenic matrix function in $\mathbb{R}^{4 n} \backslash \Gamma$, vanishing at infinity.

We will now show, by a direct calculation, that the Q-Hermitean Cauchy transform (5) can be expressed in terms of the Euclidean Cauchy type integrals

$$
C_{r, s} g(\underline{Y})=\int_{\Gamma} E_{r}(\underline{X}-\underline{Y}) \underline{n}_{s}(\underline{X}) g(\underline{X}) d S(\underline{X}), \quad \underline{Y} \notin \Gamma, r, s=0,1,2,3
$$

For this purpose, however, we need the following auxiliary result.

Lemma 1 The Euclidean Cauchy integrals (6) show the following properties in $\mathbb{R}^{4 n} \backslash \Gamma$ :

(i) $\partial_{\underline{Y}_{r}} C_{r, s} g(\underline{Y})=0, r, s=0,1,2,3$;

(ii) $\partial_{\underline{Y}_{r}} C_{s, t} g(\underline{Y})+\partial_{\underline{Y}_{s}} C_{r, t} g(\underline{Y})=0, r, s, t=0,1,2,3$.

Proof.

(i) This follows from the fact that the Cauchy kernel $E_{r}(\underline{X}-\underline{Y})$ is monogenic with respect to $\underline{Y}_{r}$ as long as $\underline{X} \neq \underline{Y}$.

(ii) A direct calculation shows that, taking into account (1),

$$
\partial_{\underline{Y}_{r}} E_{s}(\underline{X}-\underline{Y})+\partial_{\underline{Y}_{s}} E_{r}(\underline{X}-\underline{Y})=0
$$

as long as $\underline{X} \neq \underline{Y}$.

Explicitly, we then obtain for $\boldsymbol{G} \in \mathbf{C}(\boldsymbol{\Gamma})$, that

$$
\mathcal{C}[\boldsymbol{G}]=\frac{1}{4} \operatorname{circ}\left(\begin{array}{c}
C_{0,0}+C_{1,1}+C_{2,2}+C_{3,3} \\
C_{0,0}-C_{2,2}+j\left(C_{1,3}+C_{3,1}\right) \\
C_{0,0}-C_{1,1}+C_{2,2}-C_{3,3} \\
C_{0,0}-C_{2,2}-j\left(C_{1,3}+C_{3,1}\right)
\end{array}\right)[\boldsymbol{G}]
$$

In particular, for the special case of the matrix function $\boldsymbol{G}_{0}$, the action of $\mathcal{C}$ is reduced to

$$
\mathcal{C}\left[\boldsymbol{G}_{0}\right]=\frac{1}{4} \operatorname{circ}\left(\begin{array}{c}
C_{0,0} g+C_{1,1} g+C_{2,2} g+C_{3,3} g \\
C_{0,0} g-C_{2,2} g+j\left(C_{1,3} g+C_{3,1} g\right) \\
C_{0,0} g-C_{1,1} g+C_{2,2} g-C_{3,3} g \\
C_{0,0} g-C_{2,2} g-j\left(C_{1,3} g+C_{3,1} g\right)
\end{array}\right)
$$


It is clear that, in general, $\mathcal{C}\left[\boldsymbol{G}_{0}\right]$ will not turn out to be a diagonal matrix, whence its entries will not be $q$-Hermitean monogenic functions. The particular situation where $\mathcal{C}\left[\boldsymbol{G}_{0}\right]$ does become diagonal is equivalent to the conditions

$$
\begin{aligned}
C_{0,0} g & =C_{2,2} g \\
C_{1,3} g & =-C_{3,1} g \\
2 C_{0,0} g & =C_{1,1} g+C_{3,3} g
\end{aligned}
$$

whereupon

$$
\mathcal{C}\left[\boldsymbol{G}_{0}\right]=\operatorname{circ}\left(\begin{array}{c}
C_{2,2} g \\
0 \\
0 \\
0
\end{array}\right)=\operatorname{circ}\left(\begin{array}{c}
C_{0,0} g \\
0 \\
0 \\
0
\end{array}\right)=\frac{1}{2} \operatorname{circ}\left(\begin{array}{c}
C_{1,1} g+C_{3,3} g \\
0 \\
0 \\
0
\end{array}\right)
$$

At first sight, and in contrast to the complex Hermitean case, the conditions (7)(9) do not directly, i.e. without using the $\mathbf{Q}$-Hermitean monogenicity of $\mathcal{C}\left[\boldsymbol{G}_{0}\right]$, imply the $q$-Hermitean monogenicity of $C_{0,0} g$. Yet, by Lemma 1, we have that $\partial_{\underline{Y}_{0}} C_{0,0} g=0$ and, on account of (7), thus also that $\partial_{\underline{Y}_{2}} C_{0,0} g=0$ in $\Omega \backslash \Gamma$. Next, from (9) it follows that $2 \partial_{\underline{Y}_{1}} C_{0,0} g=\partial_{\underline{Y}_{1}} C_{3,3} g$, and hence, again by Lemma 1,

$$
\begin{aligned}
2 \partial_{\underline{Y}_{1}} C_{0,0} g=\partial_{\underline{Y}_{1}} C_{3,3} g & =\int_{\Gamma}\left(\partial_{\underline{Y}_{1}} E_{3}(\underline{X}-\underline{Y})\right) \underline{n}_{3}(\underline{X}) g(\underline{X}) d S(\underline{X}) \\
& =-\int_{\Gamma}\left(\partial_{\underline{Y}_{3}} E_{1}(\underline{X}-\underline{Y})\right) \underline{n}_{3}(\underline{X}) g(\underline{X}) d S(\underline{X}) \\
& =-\partial_{\underline{Y}_{3}} \int_{\Gamma} E_{1}(\underline{X}-\underline{Y}) \underline{n}_{3}(\underline{X}) g(\underline{X}) d S(\underline{X}) \\
& =-\partial_{\underline{Y}_{3}} C_{1,3} g=\partial_{\underline{Y}_{3}} C_{3,1} g=0
\end{aligned}
$$

the latter being a consequence of the second relation in (8) and the obvious $\partial_{\underline{Y}_{3}}$ monogenicity of $C_{3,1} g$. In a similar way we finally obtain that also $\partial_{\underline{Y}_{3}} C_{0,0} g=$ $\partial_{\underline{Y}_{3}} C_{1,1} g=0$, whence $C_{0,0} g$ is seen to be $q$-Hermitean monogenic.

\section{The Q-Hermitean Hilbert transform}

In this section we will first study the boundary values of the $\mathbf{Q}-$ Hermitean Cauchy integral (5). From now on, we assume the boundary $\Gamma$ to be a $(4 n-1)$-dimensional compact Liapunov surface. For further use, we also introduce the notations $\Omega^{+}=$ $\Omega$ and $\Omega^{-}=\mathbb{R}^{4 n} \backslash \Omega$.

In the following results explicit use will be made of the Hölder continuity of the normal vector to a Liapunov surface. 
Lemma 2 Let $g$ be a Hölder continuous function on $\Gamma$. Then, for $r, s=0,1,2,3$, one has

$$
\begin{aligned}
C_{r, s}^{ \pm} g(\underline{U}) & =\lim _{\Omega^{ \pm} \ni \underline{Y} \rightarrow \underline{U}} C_{r, s} g(\underline{Y}) \\
& =\int_{\Gamma} E_{r}(\underline{X}-\underline{U}) \underline{n}_{s}(\underline{X}) g(\underline{X}) d S(\underline{X}) \mp \frac{1}{2} \underline{n}_{r} \underline{n}_{s} g(\underline{U}), \quad \underline{U} \in \Gamma
\end{aligned}
$$

where the singular integral has to be understood as a Cauchy principal value.

Proof.

For $r=s$ the above formulae are nothing but the classical Plemelj-Sokhotski formulae proven by Iftimie [15]. So take $r \neq s$. Then we have, as $\underline{n}_{r}^{2}=-1$, that $C_{r, s} g=-C_{r, r} \underline{n}_{r} \underline{n}_{s} g$, and so

$$
C_{r, s}^{ \pm} g(\underline{U})=-C_{r, r}^{ \pm} \underline{n}_{r} \underline{n}_{s} g(\underline{U})
$$

Next, the Plemelj-Sokhotski formulae applied to $C_{r, r}$ show that

$$
C_{r, s}^{ \pm} g(\underline{U})=\int_{\Gamma} E_{r}(\underline{X}-\underline{U}) \underline{n}_{s}(\underline{X}) g(\underline{X}) d S(\underline{X}) \mp \frac{1}{2} \underline{n}_{r} \underline{n}_{s} g(\underline{U})
$$

Lemma 3 Let $g$ be a Hölder continuous function on $\Gamma$. Then, for $r, s=0,1,2,3$, one has

$$
\begin{aligned}
& \left(C_{r, s}+C_{s, r}\right)^{ \pm} g(\underline{U})=\lim _{\Omega^{ \pm} \ni \underline{Y} \rightarrow \underline{U}}\left(C_{r, s}+C_{s, r}\right) g(\underline{Y}) \\
& \quad=\int_{\Gamma}\left(E_{r}(\underline{X}-\underline{U}) \underline{n}_{s}(\underline{X})+E_{s}(\underline{X}-\underline{U}) \underline{n}_{r}(\underline{X})\right) g(\underline{X}) d S(\underline{X}) \pm \delta_{r, s} g(\underline{U}), \quad \underline{U} \in \Gamma
\end{aligned}
$$

where $\delta_{r, s}$ stands for the Kronecker delta.

Proof.

This is a direct consequence of Lemma 2 and of the orthogonality relations $\underline{n}_{r} \underline{n}_{s}+$ $\underline{n}_{s} \underline{n}_{r}=0$, for $r \neq s$.

Remark 1 It is worth observing how the Hölder continuity of the normal vector $\underline{n}_{r}, r=0, \ldots, 3$ was used to guarantee the existence of continuous limit values of $C_{r, s}$, as well as the validity of the Plemelj-Sokhotski formulae. When $\Gamma$ is not a Liapunov surface (but for instance an Ahlfors-David regular surface) another approach needs to be followed in order to obtain the desired results. However, we will not further develop these technical aspects here.

As a consequence of Lemma 3, we may further symmetrize relation (10) as follows. 
Corollary 1 Under the conditions (7)-(9) for the function g, the diagonal matrix $\mathcal{C}\left[\boldsymbol{G}_{0}\right]$ in (10) may be replaced by any of the matrices

$$
\operatorname{circ}\left(\begin{array}{c}
C_{r, r} g \\
0 \\
0 \\
0
\end{array}\right), \quad r=1,2,3
$$

Proof.

Indeed, as we pointed out, conditions (7)-(9) imply that, for example

$$
\partial_{\underline{Y}_{3}} C_{0,0} g=\partial_{\underline{Y}_{3}} C_{1,1} g=0, \quad \text { in } \mathbb{R}^{4 n} \backslash \Gamma
$$

In combination with Lemma 3 this implies that the function $C_{0,0} g-C_{1,1} g$, being $\partial_{\underline{Y}_{3}}$-monogenic in $\mathbb{R}^{4 n} \backslash \Gamma$, has a null jump through $\Gamma$, whence $C_{0,0} g \equiv C_{1,1} g$ in the whole of $\mathbb{R}^{4 n}$. A similar argument yields $C_{0,0} g \equiv C_{3,3} g$.

Let us now, for a circulant matrix function $\boldsymbol{G}$ defined on $\Gamma$, introduce the following matrix operator:

$$
\boldsymbol{\mathcal { H }}[\boldsymbol{G}]=\frac{1}{4} \operatorname{circ}\left(\begin{array}{c}
H_{0,0}+H_{1,1}+H_{2,2}+H_{3,3} \\
H_{0,0}-H_{2,2}+j\left(H_{1,3}+H_{3,1}\right) \\
H_{0,0}-H_{1,1}+H_{2,2}-H_{3,3} \\
H_{0,0}-H_{2,2}-j\left(H_{1,3}+H_{3,1}\right)
\end{array}\right)[\boldsymbol{G}]
$$

where

$$
H_{r, s} g(\underline{U})=2 \int_{\Gamma} E_{r}(\underline{X}-\underline{U}) \underline{n}_{s}(\underline{X}) g(\underline{X}) d S(\underline{X}), \quad \underline{U} \in \Gamma
$$

Invoking (11) and in virtue of Lemma 3, the following result is then readily obtained.

Theorem 3 Let $\boldsymbol{G} \in \mathbf{C}^{0, \alpha}(\Gamma)(0<\alpha \leq 1)$, then the continuous limit values of its $\mathbf{Q}$-Hermitean Cauchy integral $\mathcal{C}[\boldsymbol{G}]$ exist and are given by

$$
\mathcal{C}^{ \pm}[\boldsymbol{G}](\underline{U})=\frac{1}{2}(\mathcal{H}[\boldsymbol{G}](\underline{U}) \pm \boldsymbol{G}(\underline{U})), \quad \underline{U} \in \Gamma
$$

Corollary 2 Let $g \in C^{0, \alpha}(\Gamma)(0<\alpha \leq 1)$, then the continuous limit values of its $\mathbf{Q}$-Hermitean Cauchy transform $\mathcal{C}\left[\boldsymbol{G}_{0}\right]$ exist and are given by

$$
\mathcal{C}^{ \pm}\left[\boldsymbol{G}_{0}\right](\underline{U})=\frac{1}{2}\left(\boldsymbol{\mathcal { H }}\left[\boldsymbol{G}_{0}\right](\underline{U}) \pm \boldsymbol{G}_{0}(\underline{U})\right), \underline{U} \in \Gamma .
$$

The above results motivate calling the operator $\mathcal{H}$ the $\mathbf{Q}-$ Hermitean Hilbert transform. Moreover, in the following theorem, the traditional properties of a Hilbert transform are established for the operator $\mathcal{H}$.

Theorem 4 One has 
(i) $\mathcal{H}$ is a bounded linear operator on $\left(\mathbf{C}^{0, \alpha}(\Gamma),\|\bullet\|_{\alpha}\right)(0<\alpha<1)$

(ii) $\mathcal{H}$ is an involution on $\mathbf{C}^{0, \alpha}(\Gamma)(0<\alpha<1)$, that is, $\mathcal{H}^{2}=\mathcal{I}$, where $\mathcal{I}$ is the $(4 \times 4)$ identity matrix operator.

Proof.

The proof of (i) is based in the fact that all operators $H_{r, s}, r, s=0,1,2,3$, are bounded linear operators on $C^{0, \alpha}(\Gamma)(0<\alpha<1)$, see [2].

Next, by Theorem 3 we have that for $\boldsymbol{G} \in \mathbf{C}^{0, \alpha}(\Gamma)$ the function $\frac{1}{2}(\boldsymbol{H}[\boldsymbol{G}]+\boldsymbol{G})$ is the interior boundary value on $\Gamma$ of $\mathcal{C}[\boldsymbol{G}]$. The $\mathbf{Q}$-Hermitean Cauchy integral formula gives

$$
\mathcal{C}\left[\frac{1}{2}(\mathcal{H}[\boldsymbol{G}]+\boldsymbol{G})\right](\underline{Y})=\mathcal{C}[\boldsymbol{G}](\underline{Y}), \quad \underline{Y} \in \Omega^{+}
$$

or equivalently

$$
\mathcal{C}[\mathcal{H}[\boldsymbol{G}]](\underline{Y})=\mathcal{C}[\boldsymbol{G}](\underline{Y}), \underline{Y} \in \Omega^{+}
$$

Then

$$
\mathcal{C}^{+}[\mathcal{H}[\boldsymbol{G}]](\underline{U})=\mathcal{C}^{+}[\boldsymbol{G}](\underline{U}), \underline{U} \in \Gamma,
$$

which reads

$$
\frac{1}{2}(\mathcal{H}[\mathcal{H}[\boldsymbol{G}]]+\mathcal{H}[\boldsymbol{G}])=\frac{1}{2} \mathcal{H}[\boldsymbol{G}]+\frac{1}{2} \boldsymbol{G}
$$

where we have once more used Theorem 3. This clearly establishes (ii).

Theorem 3 also provides us with the possibility of defining on $\mathbf{C}^{0, \alpha}(\Gamma)$ the projection operators $\mathcal{P}$ and $\mathcal{Q}$ given by

$$
\mathcal{P}=\frac{1}{2}[\mathcal{I}+\mathcal{H}], \quad \mathcal{Q}=\frac{1}{2}[\mathcal{I}-\mathcal{H}]
$$

Then, of course, we have

$$
\mathcal{P}+\mathcal{Q}=\mathcal{I}, \mathcal{P}-\mathcal{Q}=\mathcal{H}, \mathcal{P}^{2}=\mathcal{P}, \mathcal{Q}^{2}=\mathcal{Q}, \mathcal{P} \mathcal{Q}=\mathcal{Q P}=\boldsymbol{O}
$$

This fact gives rise to the direct sum decomposition

$$
\mathbf{C}^{0, \alpha}(\Gamma)=\mathcal{P}\left[\mathbf{C}^{0, \alpha}(\Gamma)\right] \oplus \mathcal{Q}\left[\mathbf{C}^{0, \alpha}(\Gamma)\right],
$$

so that each matrix function $\boldsymbol{G} \in \mathbf{C}^{0, \alpha}(\Gamma)$ admits a unique decomposition into components belonging to $\mathcal{P}\left[\mathbf{C}^{0, \alpha}(\Gamma)\right]$ and to $\mathcal{Q}\left[\mathbf{C}^{0, \alpha}(\Gamma)\right]$, respectively.

\section{Acknowledgments}

This paper was written during a scientific stay of the first author at the Clifford Research Group of the Department of Mathematical Analysis of Ghent University, supported by a grant of the research council of Ghent University (BOF). He wishes to thank the members of the Clifford Research Group for their kind hospitality during this stay. 


\section{References}

[1] R. Abreu Blaya, J. Bory Reyes, F. Brackx, H. De Schepper, F. Sommen, Cauchy integral formulae in Hermitean quaternionic Clifford analysis, accepted for publication in Compl. Anal. Oper. Theory.

[2] R. Abreu Blaya, J. Bory Reyes, D. Peña Peña, Clifford Cauchy type integrals on Ahlfors-David regular surfaces in $\mathbb{R}^{m+1}$, Adv. Appl. Cliff. Algebras $\mathbf{1 3}$ (no.2) (2003), 133-156.

[3] F. Brackx, R. Delanghe, F. Sommen, Clifford Analysis, Research Notes in Mathematics 76, Pitman, Boston, MA, 1982.

[4] F. Brackx, J. Bureš, H. De Schepper, D. Eelbode, F. Sommen, V. Souček, Fundaments of Hermitean Clifford analysis - Part I: Complex structure, Complex Anal. Oper. Theory 1(3) (2007), 341-365.

[5] F. Brackx, J. Bureš, H. De Schepper, D. Eelbode, F. Sommen, V. Souček, Fundaments of Hermitean Clifford analysis - Part II: Splitting of hmonogenic equations, Complex Var. Elliptic Equ. 52(10-11) (2007), 10631079 .

[6] F. Brackx, H. De Schepper, F. Sommen, The Hermitian Clifford analysis toolbox, Adv. Appl. Clifford Alg. 18(3-4) (2008), 451-487.

[7] F. Brackx, B. De Knock, H. De Schepper, A matrix Hilbert transform in Hermitean Clifford analysis, J. Math. Anal. Appl. 344 (2008), 1068-1078.

[8] A. Damiano, D. Eelbode, I. Sabadini, Quaternionic Hermitian spinor systems and compatibility conditions, Adv. Geom. 11 (2011), 169-189.

[9] A. Damiano, D. Eelbode, I. Sabadini, Algebraic analysis of Hermitian monogenic functions, C.R. Acad. Sci. Paris Ser.I 346 (2008), 139-142.

[10] R. Delanghe, F. Sommen, V. Souček, Clifford Algebra and Spinor-Valued Functions, Kluwer Academic Publishers, Dordrecht, 1992.

[11] D. Eelbode, A Clifford algebraic framework for $\mathrm{sp}(\mathrm{m})$-invariant differential operators, Adv. Appl. Cliff. Algebras 17 (2007), 635-649.

[12] J. Gilbert, M. Murray, Clifford Algebras and Dirac Operators in Harmonic Analysis, Cambridge University Press, Cambridge, 1991.

[13] K. Gürlebeck, K. Habetha, W. Sprössig, Holomorphic functions in the plane and $n$-dimensional space, translated from the 2006 German original, Birkhäuser Verlag, Basel, 2008.

[14] K. Gürlebeck, W. Sprössig, Quaternionic and Clifford Calculus for Physicists and Engineers, Wiley, Chichester, 1998. 
[15] V. Iftimie, Fonctions hypercomplexes, Bull. Mathém. Soc. Sci. Mathém. Républ. Socialiste de Romanie, 9(57) (1965), 279-332.

[16] D. Peña Peña, I. Sabadini, F. Sommen, Quaternionic Clifford analysis: the Hermitian setting, Complex Anal. Oper. Theory 1 (2007), 97-113.

R. Abreu Blaya: Facultad de Informática y Matemática, Universidad de Holguín, Holguín 80100, Cuba.

E-mail: rabreu@facinf.uho.edu.cu

J. Bory Reyes: Departamento de Matemática, Universidad de Oriente, Santiago de Cuba 90500, Cuba.

E-mail: jbory@rect.uo.edu.cu

F. Brackx: Department of Mathematical Analysis, Faculty of Engineering, Ghent University, Galglaan 2, 9000 Gent, Belgium.

E-mail: fb@cage.UGent.be

H. De Shepper: Department of Mathematical Analysis, Faculty of Engineering, Ghent University, Galglaan 2, 9000 Gent, Belgium.

E-mail: hds@cage.UGent.be

F. Sommen: Department of Mathematical Analysis, Faculty of Engineering, Ghent University, Galglaan 2, 9000 Gent, Belgium.

E-mail: fs@cage.UGent.be 